“C 2018 IEEE. Personal use of this material is permitted. Permission from IEEE must be obtained for all other uses, in any current or future media, including reprinting/republishing this material for advertising or promotional purposes, creating new collective works, for resale or redistribution to servers or lists, or reuse of any copyrighted component of this work in other works." 


\title{
Performance Enhancement of an Aeroelastic Energy Harvester for Efficient Power Harvesting from Concurrent Wind Flows and Base Vibrations
}

\author{
Liya Zhao
}

\begin{abstract}
In this paper, using a high frequency mechanical stopper as a complementary energy harvester is proposed to improve the performance of energy harvesting from concurrent wind flows and base vibrations. Galloping aeroelasticity of a square-sectioned bluff body is employed to achieve limit-cycle structural oscillations. The analysis demonstrates that the bandwidth for effectively harnessing both aerodynamic and base vibratory energy is substantially widened, and simultaneously, the total power amplitude is significantly enhanced as compared to the original linear galloping energy harvester. It is concluded that the proposed system is viable solution to enhance energy conversion in situations where wind flows and base vibrations are coexisting.
\end{abstract}

\section{INTRODUCTION}

The past decades have seen an increasing interest in standalone and low-power consumption electronics such as wireless sensor networks (WSNs), data transmitters, medical implants, etc., which have found wide applications in sensing, monitoring and communication areas. A major problem is how to maintain perpetual power supply. Due to the limited lifespans of batteries, frequent replacement or recharging is required which can be cumbersome, time-consuming, and expensive, especially for those sensor nodes deployed in remote or inaccessible locations. Recently, growing efforts have been devoted to the development of energy harvesters to harness the ambient energy sources as an alternative on-site power supply. The ultimate goal is to realize automatically self-powered electronic systems such as self-powered WSNs. Available sources include wind flows, mechanical vibrations, solar energy, hydraulic power, thermal energy, and so on.

Considerable studies have been carried out to convert ambient mechanical vibrations to electricity. Various techniques have been reported to enhance the performance of base vibration piezoelectric energy harvesters in terms of broadening the bandwidth and improving the power generation efficiency $[1,2]$. Researchers have developed nonlinear energy harvesters with monostable, bistable or tristable characterstics [3] to improve the functionality of energy harvesters subjected to ambient vibrations with variant frequencies or random patterns. Frequency up-conversion techniques are also frequently exploited to harness ultra-low frequency sources [4]. Impact-based designs by introducing mechanical stoppers [5-7] are also enthusiastically pursued to harness base vibrations.

*Research supported by Seed Funding of University of Technology Sydney (No. 2232299).

Liya Zhao is with the School of Mechanical and Mechatronic Engineering, Faculty of Engineering and Information Technology,
Besides the preexisting structural vibrations, wind energy is also a ubiquitous energy source in both indoor and outdoor environments. Although large-scale wind turbines with power generation in megawatt under strong wind flow conditions are already developed very well, small-scale wind energy harvesting in milliwatt or microwatt for powering microelectronics has received limited attention until recently [8-11]. Researchers have investigated small-scale energy harvesting by exploiting various aeroelastic instabilities, such as vortex-induced vibrations (VIV) induced by periodic vortex shedding [12], modal convergent flutter of flapping airfoils [13], galloping of prisms [14-17]. Large amplitude oscillations arise from the coupling of the aerodynamic forces with the harvester structures. Considerable efforts have also been devoted to improving the efficiency of aeroelastic energy harvesters from both structural modifications [18-21] and interface circuit sophistications [17, 22].

However, all the above studies have only considered a single type of energy source, either vibration or wind flow. In many circumstances, these two sources are coexisting, such as the bridges, ships, vehicles, aircrafts, subway tunnels, etc. It is therefore worthwhile to develop energy harvesters that concurrently harness coexisting wind and vibration energy to power sensor nodes located in these situations. Bibo and Daqaq [13] were among the very first to consider energy harvesting under combined loadings. Using an aeroelastic flutter piezoelectric energy harvester under combined excitations, they found the power was enhanced as compared to that generated by two separate base vibration energy harvester and flutter energy harvester. Concurrent energy harvesting based on galloping energy harvesters and VIV energy harvesters have also been recently investigated [23, 24]. However, these concurrent energy harvesters suffer from a main drawback that enhanced power is only achieved within a very narrow bandwidth close to the resonance. When the base vibration frequency deviates from the resonance, quasiperiodic oscillations occur due to the interaction between the coexisting base vibratory and aerodynamic frequencies. There are quite few studies on enhancing the functionality of aeroelastic energy harvesters under concurrent base vibratory and aerodynamic excitations. Recently, Zhao and Yang [19] reported a novel impact-based design of concurrent base vibration and wind energy harvester with broadened bandwidth by utilizing a mechanical stopper. Electrical energy was collected from the galloping piezoelectric energy harvester. The quasi-periodic oscillations were converted to

University of Technology Sydney, 15 Broadway Ultimo 2007, NSW, Australia (phone: +61 (02) 9514 3184; e-mail: liya.zhao@uts.edu.au). 
periodic vibrations by the introduced mechanical stopper, and the coexisting excitation frequencies were forced to lock into each other over an extended frequency bandwidth.

This paper investigates the power enhancing performance of a broadband concurrent wind and base vibration energy harvester with piecewise linear restoring force and frequency up-conversion characteristic. Galloping aeroelasticity of a square-sectioned bluff body is employed to achieve limit-cycle structural oscillations. The high frequency mechanical stopper is utilized as a complementary piezoelectric energy harvester, which simultaneously broadens the operational frequency bandwidth and augments the power amplitude. Such system is proposed for the first time in the field of concurrent wind and base vibration energy harvesting.

\section{BROADBAND CONCURRENT WIND AND BASE VIBRATION ENERGY HARVESTING SYSTEM}
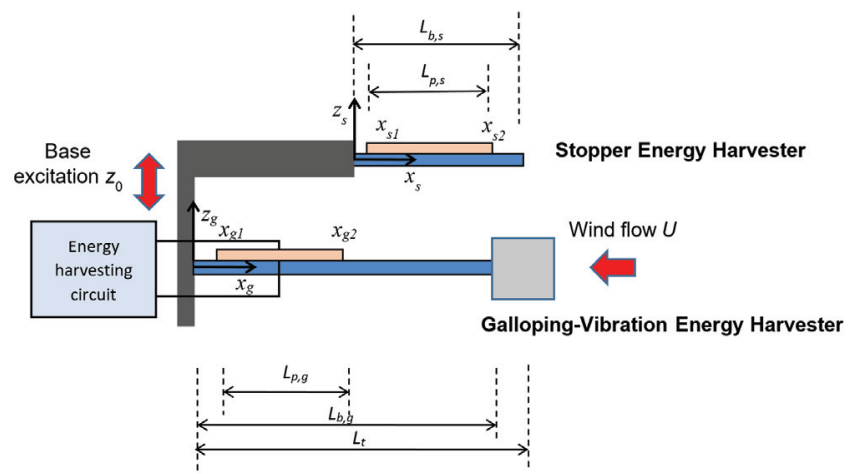

(a)

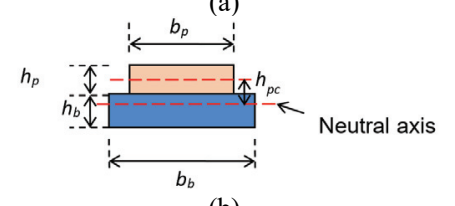

(b)

Figure. 1 Schematic of (a) the enhanced broadband wind and base vibration energy harvester system and (b) the cross section of the two piezoelectric beams

The configuration of the energy harvester under investigation is shown in Figure 1. A square-sectioned bluff body is connected to the free end of a cantilever in a way that one flat surface is facing the incoming wind flows. At this angle of attack the Den Hartog criterion of galloping is satisfied [25], and large amplitude oscillation normal to the incoming flow occurs when the wind speed $U$ exceeds the cutin speed $U_{c r}$. Galloping aeroelasticity has the characteristics of being self-excited and self-limiting, with the capability of oscillating in an infinite range of wind speeds [26], which maks it a superb candidate for the purpose of wind energy harvesting. A range of cross section geometries is able to initiate galloping. According to our previous experimental study [16], the square section performs best for wind energy harvesting in laminar flows, thus is utilized here in the proposed system. A piezoelectric laminate is bonded to the cantilever near the fixed area, where the largest strain appears. During galloping, oscillatory bending of the cantilever induces periodic alternating strain in the piezoelectric material, which is converted into electrical charges and further extracted by the energy harvesting interface circuit. The fixed end of the harvester is subjected to base vibratory excitation $z_{0}$. This harvester under combined loadings is denoted as Galloping-Vibration Energy Harvester (GVEH).

Above the GVEH, a mechanical stopper is installed on the same supporting frame. The distance between the stopper beam and the top surface of the bluff body of GVEH is $D$. The fundamental frequency of the stopper is chosen to be much higher than the GVEH. When the displacement of the bluff body of GVEH relative to the base excitation is larger than $D$, it impacts the stopper. As a result, the total restoring force will suddenly increase. This piecewise linear effective stiffness is expected to introduce hardening nonlinear behavior and a broadband response. The stopper beam continues to oscillate at its own high frequency after the bluff body departs from it. Due to the high frequency nature, the induced alternating strain on the stopper is a prospective source for energy harvesting. By attaching a second piece of piezoelectric laminate onto the stopper near the root area, this part of strain can be beneficially harnessed, making the stopper a complementary energy harvester which is denoted as Stopper Energy Harvester (SEH).

\section{AnAlytical Model}

Multi-way coupling behaviors exist in an aeroelastic piezoelectric energy harvesting system, i.e., the aero-electromechanical coupling between the harvester structure, air flow, piezoelectric transducer and electric circuit. To fully evaluate the mutual coupling effects, the analytical model should incorporate two parts: the electromechanical model and the aerodynamic model $[10,15-17,22,23,27]$. It has been validated in our previous experimental study that a galloping piezoelectric energy harvester always operates at its first vibration mode $[16,18]$. The range of base vibration frequency of interest in this study is in the neighborhood of the fundamental frequency of the GVEH. Except for the instants when the impacts happen, the SEH undergoes free oscillation at its own fundamental frequency. Therefore, it is safe to establish the coupled single-mode distributed parameter model as

$$
\begin{gathered}
\ddot{\eta}_{g}(t)+2 \zeta_{g} \omega_{n 1} \dot{\eta}_{g}(t)+\omega_{n 1}{ }^{2} \eta_{g}(t)+\chi_{g} V_{1}(t)=f_{g, \text { base }}(t)+f_{\text {galloping }}(t) \\
\ddot{\eta}_{s}(t)+2 \zeta_{s} \omega_{n 2} \dot{\eta}_{s}(t)+\omega_{n 2}{ }^{2} \eta_{s}(t)+\chi_{s} V_{2}(t)=f_{s, \text { base }}(t) \\
I_{1}(t)+C_{p, g} \dot{V}_{1}(t)-\chi_{g} \dot{\eta}_{g}(t)=0 \\
I_{2}(t)+C_{p, s} \dot{V}_{2}(t)-\chi_{s} \dot{\eta}_{s}(t)=0
\end{gathered}
$$

where the subscripts $g$ and $s$ represent the $G V E H$ and $S E H$, respectively; $\eta(t)$ is the modal coordinate; $I(t)$ and $V(t)$ are the outgoing current and voltage on the piezoelectric transducer; $\zeta$ is the mechanical damping ratio; $C_{p}$ is the piezoelectric capacitance; $\chi$ is the modal electromechanical coupling coefficient; $\omega_{n 1}$ and $\omega_{n 2}$ are the fundamental frequencies of the GVEH and SEH, respectively; and $f_{\text {base }}(t)$ and $f_{\text {galloping }}(t)$ are respectively the base excitation induced modal inertial force and galloping induced modal aerodynamic force. $\chi$ is calculated as $\chi=-E_{p} d_{31} b_{p} h_{p c}\left[\phi^{\prime}\left(x_{2}\right)-\phi^{\prime}\left(x_{1}\right)\right]$, where $E_{p}, d_{31}, b_{p}, h_{p c}$, $\phi^{\prime}\left(x_{2}\right)$ and $\phi^{\prime}\left(x_{1}\right)$ are the piezoelectric elastic modulus, piezoelectric constant, width of the piezoelectric sheet, position of the center of the piezoelectric sheet with respect to the neutral axis of the composite cross section, and the slopes of first vibration mode at the end and start points of the piezoelectric sheet along the cantilever, respectively. Usually, $x_{1}$ is taken to be as small as possible to ensure a large strain of 
the piezoelectric sheet. The mode shape is mass normalized. The modal forces are related to the base vibratory excitation amplitude $z_{0}$, wind speed $U$, the respective distributed mass $m$ and first vibration mode $\phi(x)$ of the GVEH and SEH, the mass of the bluff body $M_{t}$, and the slope of first vibration mode at the tip of the GVEH $\phi^{\prime}\left(L_{t}\right)$. They are given by

$$
\begin{aligned}
& f_{g, \text { base }}(t)=-\left[\int_{0}^{L_{b, g}} \phi_{g}\left(x_{g}\right) m_{g} d x_{g}+\phi_{g}\left(L_{t}\right) M_{t}\right] \ddot{z}_{0}(t) \\
& f_{s, \text { base }}(t)=-\left[\int_{0}^{L_{b, s}} \phi_{s}\left(x_{s}\right) m_{s} d x_{s}\right] \ddot{z}_{0}(t) \\
& f_{\text {galloping }}(t)=\phi_{g}\left(L_{t}\right) \times \frac{1}{2} \rho h L U^{2} \sum_{i=1}^{3} A_{i} \alpha^{i} \\
& =\phi_{g}\left(L_{t}\right) \times \frac{1}{2} \rho h L U^{2} \sum_{i=1}^{3} A_{i}\left[\frac{\phi_{g}\left(L_{t}\right) \dot{\eta}_{g}(t)+\dot{z}_{0}(t)}{U}+\phi_{g}^{\prime}\left(L_{t}\right) \eta_{g}(t)\right]^{i}
\end{aligned}
$$

where $\rho, h, L, A_{i}$ and $\alpha$ are, respectively, the air density, frontal dimension and length of the bluff body, empirical aerodynamic coefficients and angle of attack. Here, the aerodynamic force in (7) is represented based on the quasisteady hypothesis $[14,26]$. This representation has been validated experimentally in previous studies to be sufficiently accurate for the prediction of the mechanical and electrical responses of a galloping piezoelectric energy harvester [16, 18]. (1)-(4) are the distributed parameter model for the case when the displacement of the bluff body is smaller than $D$. For the convenience of formulation and calculation, (1)-(4) are able to be degraded into the form of a lumped parameter model (Fig.2) by letting

$$
\begin{gathered}
M_{g}=\frac{1}{\left[\phi_{g}\left(L_{t}\right)\right]^{2}}, C_{g}=\frac{2 \zeta_{g} \omega_{n 1}}{\left[\phi_{g}\left(L_{t}\right)\right]^{2}}, K_{g}=\frac{\omega_{n 1}^{2}}{\left[\phi_{g}\left(L_{t}\right)\right]^{2}}, \Theta_{g}=\frac{\chi_{g}}{\phi_{g}\left(L_{t}\right)}, \\
M_{s}=\frac{1}{\left[\phi_{s}\left(L_{b, s}\right)\right]^{2}}, C_{s}=\frac{2 \zeta_{s} \omega_{n 2}}{\left[\phi_{s}\left(L_{b, s}\right)\right]^{2}}, K_{s}=\frac{\omega_{n 2}{ }^{2}}{\left[\phi_{s}\left(L_{b, s}\right)\right]^{2}}, \Theta_{s}=\frac{\chi_{s}}{\phi_{s}\left(L_{b, s}\right)}, \\
u_{1}(t)=\eta_{g}(t) \phi_{g}\left(L_{t}\right), u_{2}(t)=\eta_{s}(t) \phi_{s}\left(L_{b, s}\right) \\
\lambda_{1}=\phi_{h}\left(L_{t}\right)\left[\int_{0}^{L_{b}} \phi_{h}\left(x_{h}\right) m_{h} d x_{h}+\phi_{h}\left(L_{t}\right) M_{t}\right] \\
\lambda_{2}=\phi_{s}\left(L_{s}\right)\left[\int_{0}^{L_{s}} \phi_{s}\left(x_{s}\right) m_{s} d x_{s}\right] \\
\beta=\frac{\phi_{g}^{\prime}\left(L_{t}\right)}{\phi_{g}\left(L_{t}\right)}
\end{gathered}
$$

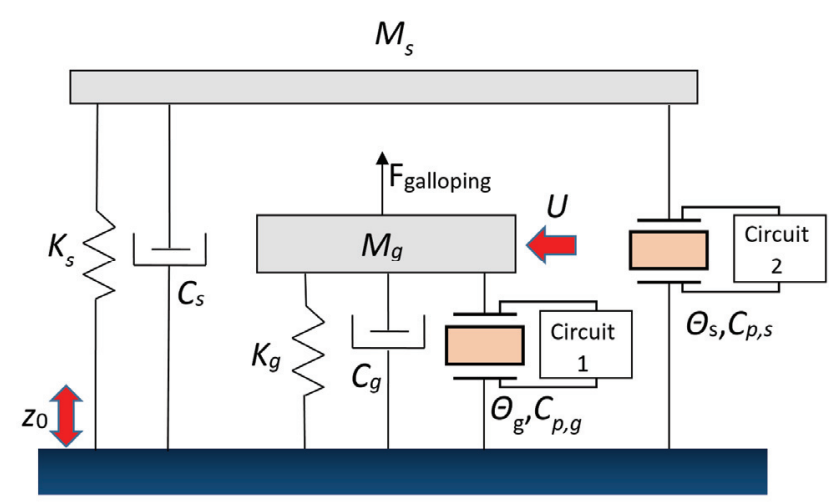

Figure. 2 Schematic of lumped parameter model

where $M, C, K$ and $\Theta$ are the effective mass, damping, stiffness and lumped electromechanical coupling of the GVEH and $\mathrm{SEH}$; and $u_{1}(t)$ and $u_{2}(t)$ are the transverse displacements at the tip. Further introducing $\lambda_{1}, \lambda_{2}$ and $\beta$ to represent the inertial forcing correction factors of the two harvesters and coefficient of rotation deformation of the bluff body given in (16)-(18), (1)-(4) can be rearranged as

$$
\begin{aligned}
& \left\{\begin{array}{l}
M_{g} \ddot{u}_{1}(t)+C_{g} \dot{u}_{1}(t)+K_{g} u_{1}(t)+\Theta_{g} V_{1}(t) \\
=-\lambda_{1} M_{g} \ddot{z}_{0}(t)+\frac{1}{2} \rho h L U^{2} \sum_{i=1}^{3}\left[A_{i}\left(\frac{\dot{u}_{1}(t)+\dot{z}_{0}(t)}{U}+\beta u_{1}(t)\right)\right]^{i} \\
M_{s} \ddot{u}_{2}(t)+C_{s} \dot{u}_{2}(t)+K_{s} u_{2}(t)+\Theta_{s} V_{2}(t)=-\lambda_{2} M_{s} \ddot{z}_{0}(t) \\
I_{1}(t)+C_{p, g} \dot{V}_{1}(t)-\Theta_{g} u_{1}(t)=0 \\
I_{2}(t)+C_{p, s} \dot{V}_{2}(t)-\Theta_{s} u_{2}(t)=0
\end{array}\right. \\
& \left(u_{1}(t)<D\right)
\end{aligned}
$$

When the impacts happen, the GVEH and SEH engage each other. For simplicity, the collision is regarded to be completely elastic. The coupled model becomes

$$
\begin{aligned}
& \left\{\begin{array}{l}
\left(M_{g}+M_{s}\right) \ddot{u}_{1}(t)+\left(C_{g}+C_{s}\right) \dot{u}_{1}(t)+\left(K_{g}+K_{s}\right) u_{1}(t)-K_{s} D+\Theta_{g} V_{1}(t)+\Theta_{s} V_{2}(t) \\
=-\left(\lambda_{1} M_{g}+\lambda_{2} M_{s}\right) \ddot{z}_{0}(t)+\frac{1}{2} \rho h L U^{2} \sum_{i=1}^{3}\left[A_{i}\left(\frac{\dot{u}_{1}(t)+\dot{z}_{0}(t)}{U}+\beta u_{1}(t)\right)\right]^{i} \\
u_{2}(t)=u_{1}(t)-D \\
I_{1}(t)+C_{p, g} \dot{V}_{1}(t)-\Theta_{g} u_{1}(t)=0 \\
I_{2}(t)+C_{p, s} \dot{V}_{2}(t)-\Theta_{s} u_{2}(t)=0
\end{array}\right. \\
& \left(\begin{array}{l}
\left.u_{1}(t) \geq D\right)
\end{array}\right.
\end{aligned}
$$

\section{RESUlTS AND DisCUSSION}

This section presents a case study based on the established aero-electro-mechanically coupled model. The performance of the proposed design of a broadband concurrent wind and base vibration energy harvester with piezoelectric transduction and galloping instability is investigated. It is worth mentioning that using a fabricated prototype with a rigid mechanical stopper without piezoelectric coupling, the predicted power output responses of an impact engaged piezoelectric GVEH using a similar single-mode model have been experimentally validated in a previous study [19]. The benefits of the modified design is that by utilizing the high frequency stopper as a complementary energy harvester, the broadband performance is achieved simultaneously with a tremendous power level enhancement. The properties of the energy harvesting system used in the simulations are listed in Table 1.

TABLE I. PROPERTIES OF THE GALLOPING-VIBRATION-STOPPER ENERGY HARVESTER TABLE TYPE STYLES

\begin{tabular}{|l|l|}
\hline \multicolumn{1}{|c|}{ Properties } & \multicolumn{1}{|c|}{ Values } \\
\hline Galloping harvester effective mass $M_{g}(\mathrm{~g})$ & 2.78 \\
Stopper harvester effective mass $M_{s}(\mathrm{~g})$ & 0.44 \\
Galloping harvester effective stiffness $K_{g}\left(\mathrm{Nm}^{-1}\right)$ & 31.56 \\
Stopper harvester effective stiffness $K_{s}\left(\mathrm{Nm}^{-1}\right)$ & 520.62 \\
Galloping harvester damping $C_{g}\left(\mathrm{Nsm}^{-1}\right)$ & 0.00652 \\
Stopper harvester damping $C_{s}\left(\mathrm{Nsm}^{-1}\right)$ & 0.01054 \\
Galloping harvester electromechanical coupling & $9.599 \times 10^{-5}$ \\
coefficient $\Theta_{g}\left(\mathrm{NV}^{-1}\right)$ & \\
Stopper harvester electromechanical coupling & $6.408 \times 10^{-4}$ \\
coefficient $\Theta_{s}\left(\mathrm{NV}^{-1}\right)$ & 25.7 \\
Galloping harvester piezoelectric capacitance $C_{p, g}(\mathrm{nF})$ & 25.7 \\
Stopper harvester piezoelectric capacitance $C_{p, s}(\mathrm{nF})$ & $\mathrm{Square}$ \\
Cross section shape of bluff body & $20 \times 20$ \\
Cross section dimension of bluff body $\left(\mathrm{mm}^{2}\right)$ & 100 \\
Length of bluff body $L(\mathrm{~mm})$ & 1.204 \\
Air density $\rho$ at $\left.20^{\circ} \mathrm{C}(\mathrm{kgm})^{-3}\right)$ & $2.3,0,-18$ \\
Aerodynamic coefficients $A_{1} \sim A_{3}$ & \\
\hline
\end{tabular}


The aerodynamic empirical coefficients $A_{T} \sim A_{3}$ for the square-sectioned bluff body are taken as $A_{1}=2.3, A_{2}=0$, and $A_{3}=-18$ [14]. Here, $A_{1}$ being positive ensures that the crosssection of the bluff body satisfies the Den Hartog criterion and is able to be self-excited into galloping oscillations, while $A_{3}$ being negative brings a positive nonlinear aerodynamic damping into the vibration system, achieving a steady-state limit-cycle galloping oscillation at each wind speed. Advanced energy harvesting circuits should be used to rectify and regulate the electrical output during the wind energy harvesting [17, 22]. In this study, the focus in on the conversion of wind and base vibration energy into electricity, and the power regulation and storage are not considered. Therefore, a simple AC circuit consisting of a pure resistive load $R$ is employed. The average power $P_{\text {ave }}$ is calculated as $P_{\text {ave }}=V_{R M S}{ }^{2} / R$ where $V_{R M S}$ is the RMS value of $\mathrm{V}$.

The respective optimal resistive load for the harvesters are determined first. The variation of $P_{\text {ave }}$ with $R$ is calculated for the GVEH at a fixed wind speed $U$ of $4 \mathrm{~m} / \mathrm{s}$ with no base excitation, and optimal load is found to be $333 \mathrm{k} \Omega$ where the maximum power is obtained. As for the SEH, the optimal load is determined to be $21 \mathrm{k} \Omega$ from the response of $P_{\text {ave }}$ versus $R$ at its fundamental frequency of $172.9 \mathrm{~Hz}$ with a base acceleration $a_{0}$ (i.e., $\left|\ddot{z}_{0}\right|$ ) of $1 \mathrm{~m} / \mathrm{s}^{2}$. The optimal load of the $\mathrm{SEH}$ is much smaller than that of the GVEH. This is reasonable and can be deduced by the fact that the fundamental frequency $\omega_{n 2}$ is much higher than $\omega_{n 1}$ while the capacitance $C_{p, g}$ and $C_{p, s}$ are equal, since the optimal load can be approximated as $1 /\left(\omega_{n} C_{p}\right)$. In the following study, the respective optimal loads are utilized for the response calculations of the two harvesters. For the purpose of comparison, the performance of a linear GVEH in the absence of a mechanical stopper is first considered. With the optimal load resistance, when subjected to pure wind flows, the variation of $P_{\text {ave }}$ of the GVEH as a function of $U$ is shown in Fig. 3(a). It is seen that the GVEH starts to extract wind energy when $U$ exceeds $3 \mathrm{~m} / \mathrm{s}$, which is the cut-in wind speed $U_{c r}$. Beyond $U_{c r}, P_{\text {ave }}$ steadily increases with the increase of $U$. Figure 3(b) shows the variation of $P_{\text {ave }}$ of the GVEH with the base vibration frequency $\omega$ under pure base excitations of $a_{0}=1 \mathrm{~m} / \mathrm{s}^{2}$. It is seen that the response is linear with a narrow bandwidth around the fundamental frequency $\omega_{n 1}$ of $16.9 \mathrm{~Hz}$, where the maximum $P_{\text {ave }}$ achieved. Considering the condition with combined aerodynamic and base vibratory excitations, the variation of $P_{\text {ave }}$ of the GVEH with $\omega$ is plotted in Fig. 3(c), where $U$ is constant at $5 \mathrm{~m} / \mathrm{s}$ and $a_{0}$ is constant at $1 \mathrm{~m} / \mathrm{s}^{2}$. It is noted that when $\omega$ is away from the resonance, $P_{\text {ave }}$ under the concurrent excitations is constant at $1.2 \mathrm{~mW}$, which is found to be almost the same with that under the pure galloping excitations by inspecting Fig. 3(a). When $\omega$ sweeps up to be close to the resonance, $P_{\text {ave }}$ dramatically drops to a minimum value. The maximum $P_{\text {ave }}$ of $1.8 \mathrm{~mW}$ is achieved around the resonance. Finally, with the further increase of $\omega, P_{\text {ave }}$ drops back to the level under the pure galloping excitations. In this paper, we define the bandwidth of concurrent energy harvesting to be the frequency range within which both the aerodynamic and base vibratory inputs are effectively harnessed, i.e., when $P_{\text {ave }}$ is larger than that from pure galloping or pure base vibration excitation. The response of $P_{\text {ave }}$ for the linear GVEH indicates that, only in the region where the base excitation frequency is very close to the resonance, the two types of excitations can supplement each other and are both effectively harnessed. Outside of this narrow bandwidth, the base vibration does not contribute to the energy harvesting process. Actually, a further look at the time domain response of the tip displacement of the GVEH at $17.5 \mathrm{~Hz}$ which is slightly over the resonance given in Fig. 3(d)

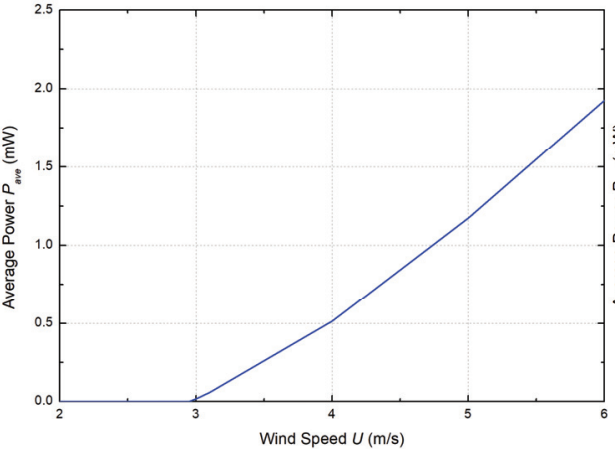

(a)

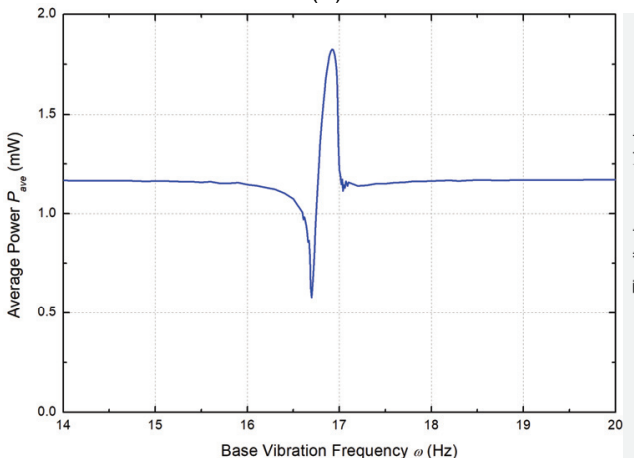

(c)

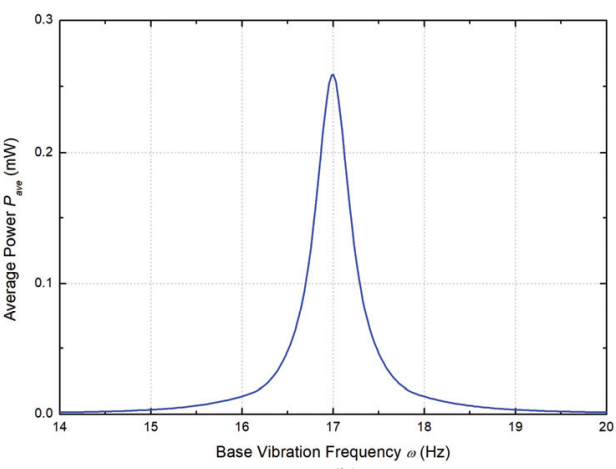

(b)

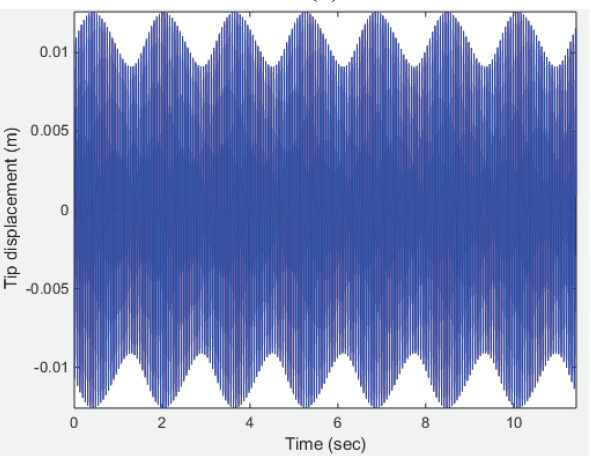

(d)

Figure 3. Responses of the original linear GVEH (a) $P_{\text {ave }}$ versus $U$ under pure galloping, (b) $P_{\text {ave }}$ versus $\omega$ under pure base vibration, (c) $P_{\text {ave }}$ versus $\omega$ under concurrent excitations and (d) tip displacement in time domain. 
shows that, due to the coexistence of two excitation frequencies, the harvester undergoes quasi-periodic oscillations with an amplitude modulation. As a result, the peak displacement amplitude is $12.6 \mathrm{~mm}$, while the RMS displacement is only $7.8 \mathrm{~mm}, 12.5 \%$ lower than that of a fully excited harvester undergoing periodic oscillations with the same peak displacement amplitude. This reveals another disadvantage of a linear GVEH besides the narrow bandwidth, that is, the structure endures large deflection outside the resonance yet fails to effectively convert the strain energy [19].

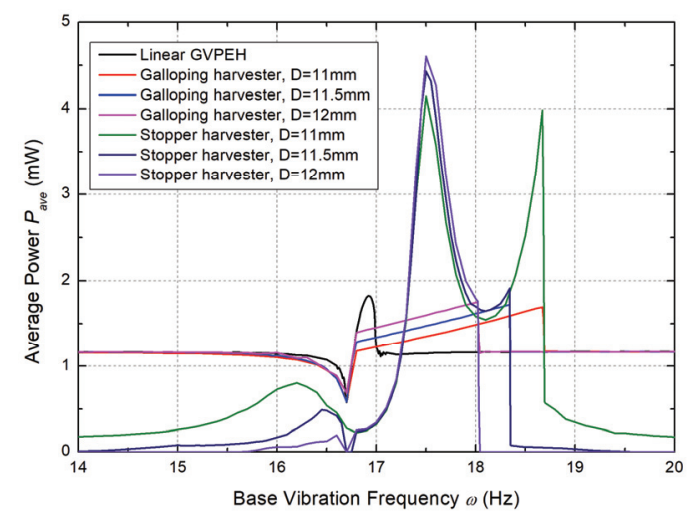

(a)

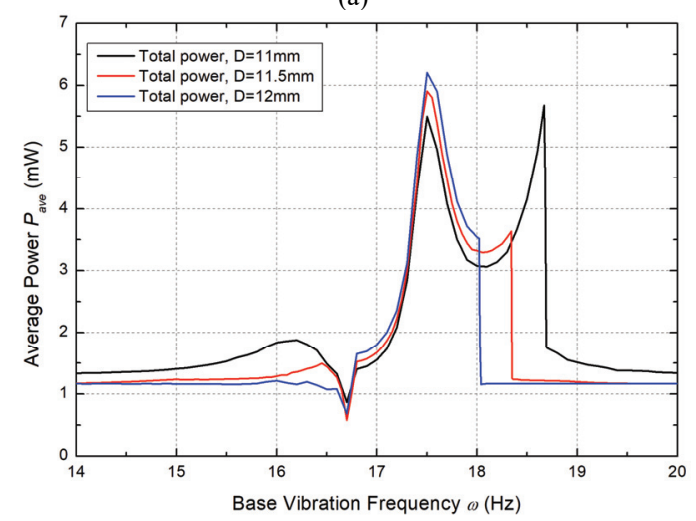

(b)

Figure 4. Variation of (a) power of the GVEH and the SEH and (b) total power of the integrated system with base vibration frequency

By employing a mechanical stopper to add piecewise linearity and at the same time operate as a complementary energy harvester, the variation of $P_{\text {ave }}$ for the GVEH and SEH with $\omega$ at different configurations of GVEH-SEH distance $D$ is depicted in Fig. 4(a). $U$ and $a_{0}$ are still constant at $5 \mathrm{~m} / \mathrm{s}$ and $1 \mathrm{~m} / \mathrm{s}^{2}$, respectively. It is seen that for the GVEH, when $\omega$ sweeps up to be close to the resonance, the difference between the displacements of the GVEH and SEH reaches $D$, and as a result, the GVEH starts to impact the SEH and experience a sudden increase in the effective stiffness during each oscillation cycle. The bandwidth of the GVEH is significantly extended beyond the resonance for all three values of $D$, within which $P_{\text {ave }}$ steadily increases. For example, with $\mathrm{D}=11 \mathrm{~mm}$, $P_{\text {ave }}$ of the GVEH steadily increases from 1.2 to $1.6 \mathrm{~mW}$ within a frequency range from 16.8 to $18.7 \mathrm{~Hz}$. Increasing $D$ results in a decrease in the bandwidth and an increase in the power amplitude. As for the SEH, it is noted that with the increase of $\omega, P_{\text {ave }}$ gradually increases to a local maximum, then drops when $\omega$ increases to approach the resonance of the GVEH. Once $\omega$ sweeps up to be over the resonance, $P_{\text {ave }}$ of the SEH quickly increases to another peak, which is greatly higher than that of the piecewise linear GVEH or the original linear GVEH. The maximum $P_{\text {ave }}$ over the whole range of frequencies of interest is achieved at this second peak for all three considered $D$. Subsequently, $P_{\text {ave }}$ decreases with $\omega$. When $\omega$ increases to the point where the GVEH stops engaging the SEH, with a larger $D$ of $12 \mathrm{~mm}, P_{\text {ave }}$ of the SEH directly drops back to the level generated from pure galloping oscillations. In contrast, for the cases where $D$ is smaller at $11.5 \mathrm{~mm}$ and $11 \mathrm{~mm}, P_{\text {ave }}$ of the $\mathrm{SEH}$ increases to a third peak before it drops to the pure galloping level. The amplitude of the third peak of $P_{\text {ave }}$ $(4.0 \mathrm{~mW})$ is comparable to that of the second peak $(4.1 \mathrm{~mW})$ when $D$ is $11 \mathrm{~mm}$.

Combining the power output from the GVEH and SEH, the variation of the total average power of the integrated concurrent energy harvesting system as function of the base vibration frequency is shown in Fig. 4(b). It is seen that as compared to the original linear GVEH (Fig. 3(c)), a substantial extension in the bandwidth is achieved simultaneously with a significant enhancement of the power amplitude. As an example, with $D=11 \mathrm{~mm}$, the bandwidth is $1.9 \mathrm{~Hz}(16.8-$ $18.7 \mathrm{~Hz}$ ) and the maximum power is $5.5 \mathrm{~mW}$, corresponding to 8.5 and 2.0 times increase when compared to the original linear GVEH $(0.2 \mathrm{~Hz}$ bandwidth from 16.8 to $17.0 \mathrm{~Hz}$ and $1.8 \mathrm{~mW}$ maximum power). It is worth mentioning that the piezoelectric materials are doubled in the integrated system as compared to the original GVEH. Considering the power density per volume of piezoelectric materials, the power enhancement is still ensured with at least $50 \%$ increase. A qualitative explanation of the high power output of the SEH can be that with the same composite cross section geometry and piezoelectric sheet with those of the GVEH, the slope of the first mode shape at the same position $x_{2}$, i.e., $\phi^{\prime}\left(x_{2}\right)$, of the SEH will be much larger than that of the GVEH since the cantilever length of the former is taken to be only the half of the latter (Fig. 1(a)) $\phi(x)$ of the two beams are both mass normalized. As a result, the electromechanical coupling of the SEH is much higher than

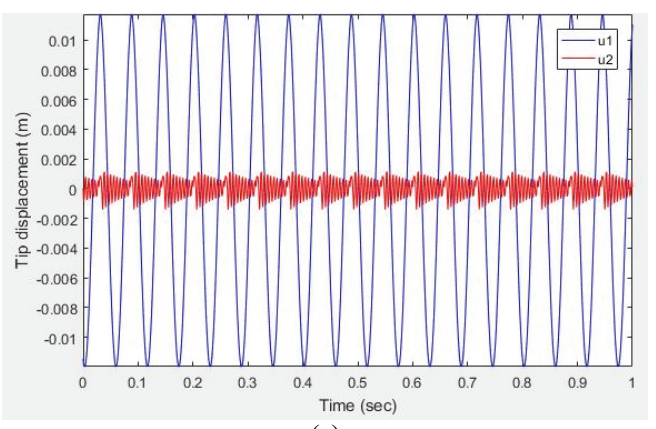

(a)

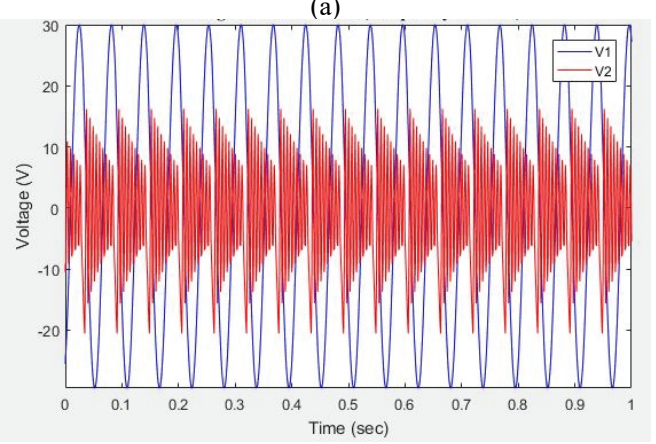

(b)

Figure 5. Time domain responses of (a) tip displacement of the GVEH $u_{1}$ and SHE $u_{2}$, and (b) voltage of the GVEH $V_{1}$ and SEH $V_{2}$ 
that of the GVEH (Table 1). At the same time, the high frequency oscillation of the $\mathrm{SEH}$ is more beneficial for the energy conversion since the power is roughly estimated to be proportional to the cube of the vibration frequency.

The steady state response of the tip displacement in time domain for the GVEH and SEH at the base vibration frequency of $17.5 \mathrm{~Hz}$ is shown in Fig. $5(\mathrm{a})$, with $U=5 \mathrm{~m} / \mathrm{s}$ and $a_{0}=1 \mathrm{~m} / \mathrm{s}^{2}$. It is seen clearly that during each vibration cycle, the GVEH impacts the SEH, and leads to a high frequency self-oscillation of the SEH. In this way, the wind energy captured by the bluff body of the GVEH is transferred to the high frequency SEH. The corresponding voltage response is shown in Fig. 5(b). High frequency voltage is generated by the $\mathrm{SEH}$, with the RMS voltage being $9.33 \mathrm{~V}$. Although the RMS voltage of the $\mathrm{SEH}$ is lower than that of the GVEH which is $21.22 \mathrm{~V}$, as mentioned before, the optimal load of the $\mathrm{SEH}$ as $21 \mathrm{k} \Omega$ is much smaller than that of the GVEH as $333 \mathrm{k} \Omega$, making the final power output of the $\mathrm{SEH}$ as $4.14 \mathrm{~mW}$ which is $200 \%$ higher than that of the GVEH as $1.35 \mathrm{~mW}$.

\section{CONCLUSION}

The potential of using a high frequency mechanical stopper as a complementary energy harvester to improve concurrent wind and base vibration energy harvesting is investigated. The wind energy is captured by the integrated energy harvesting system by exploring galloping instability of the square-sectioned bluff body of the GVEH. A single-mode aero-electro-mechanically coupled model is established which incorporates the mutual coupling effects and impact behaviors between the GVEH and SEH. The aerodynamic force is modeled based on the quasi-steady hypothesis. The analysis demonstrates that by employing a mechanical stopper to add piecewise linearity and at the same time operate as a complementary energy harvester, the bandwidth for effectively harnessing both aerodynamic and base vibratory energy is substantially widened, and simultaneously, the total power amplitude is significantly enhanced. As compared to the original linear galloping energy harvester, an 8.5 times increase in the bandwidth and a 2.0 times amplification in the output power amplitude are achieved at a wind speed of $5 \mathrm{~m} / \mathrm{s}$, an base acceleration of $1 \mathrm{~m} / \mathrm{s}^{2}$ and a GVEH-SEH distance of $11 \mathrm{~mm}$. It is concluded that adding a mechanical stopper as a complementary energy harvester to a galloping energy harvesting system is a viable solution to enhance the energy conversion performance in situations where wind flows and base vibrations are coexisting.

\section{REFERENCES}

[1] L. Tang, Y. Yang, and C. K. Soh, "Toward broadband vibration-based energy harvesting," Journal of intelligent material systems and structures, vol. 21, pp. 1867-1897, 2010.

[2] R. Harne and K. Wang, "A review of the recent research on vibration energy harvesting via bistable systems," Smart Materials and Structures, vol. 22, p. 023001, 2013.

[3] S. Zhou, J. Cao, A. Erturk, and J. Lin, "Enhanced broadband piezoelectric energy harvesting using rotatable magnets," Applied Physics Letters, vol. 102, p. 173901, 2013.

[4] H. Fu and E. M. Yeatman, "A methodology for low-speed broadband rotational energy harvesting using piezoelectric transduction and frequency up-conversion," Energy, vol. 125, pp. 152-161, 2017.

[5] M. Soliman, E. Abdel-Rahman, E. El-Saadany, and R. Mansour, "A wideband vibration-based energy harvester," Journal of Micromechanics and Microengineering, vol. 18, p. 115021, 2008.
[6] H. Liu, C. J. Tay, C. Quan, T. Kobayashi, and C. Lee, "A scrape-through piezoelectric MEMS energy harvester with frequency broadband and up-conversion behaviors," Microsystem technologies, vol. 17, pp. 17471754,2011

[7] X. Wang, C. Chen, N. Wang, H. San, Y. Yu, E. Halvorsen, et al., "A frequency and bandwidth tunable piezoelectric vibration energy harvester using multiple nonlinear techniques," Applied Energy, vol. 190, pp. 368-375, 2017.

[8] D. Li, Y. Wu, A. Da Ronch, and J. Xiang, "Energy harvesting by means of flow-induced vibrations on aerospace vehicles," Progress in Aerospace Sciences, vol. 86, pp. 28-62, 2016.

[9] J. McCarthy, S. Watkins, A. Deivasigamani, and S. John, "Fluttering energy harvesters in the wind: A review," Journal of Sound and Vibration, vol. 361, pp. 355-377, 2016.

[10] L. Zhao and Y. Yang, "On the modeling methods of small-scale piezoelectric wind energy harvesting," Smart Structures and Systems, vol. 19, pp. 67-90, 2017.

[11] L. Zhao and Y. Yang, "Toward Small-Scale Wind Energy Harvesting: Design, Enhancement, Performance Comparison, and Applicability," Shock and Vibration, vol. 2017, p. 3585972, 2017.

[12] H. D. Akaydin, N. Elvin, and Y. Andreopoulos, "The performance of a self-excited fluidic energy harvester," Smart Materials and Structures, vol. 21, p. 025007, 2012.

[13] A. Bibo and M. F. Daqaq, "Investigation of concurrent energy harvesting from ambient vibrations and wind using a single piezoelectric generator," Applied Physics Letters, vol. 102, p. 243904, 2013.

[14] A. Barrero-Gil, G. Alonso, and A. Sanz-Andres, "Energy harvesting from transverse galloping," Journal of Sound and Vibration, vol. 329, pp. 2873-2883, 2010.

[15] A. Abdelkefi, J. M. Scanlon, E. McDowell, and M. R. Hajj, "Performance enhancement of piezoelectric energy harvesters from wake galloping," Applied Physics Letters, vol. 103, p. 033903, 2013.

[16] L. Zhao, L. Tang, and Y. Yang, "Comparison of modeling methods and parametric study for a piezoelectric wind energy harvester," Smart Materials and Structures, vol. 22, p. 125003, 2013.

[17] L. Zhao, L. Tang, J. Liang, and Y. Yang, "Synergy of wind energy harvesting and synchronized switch harvesting interface circuit," IEEE/ASME Transactions on Mechatronics, vol. 22, pp. 1093-1103, 2017.

[18] L. Zhao and Y. Yang, "Enhanced aeroelastic energy harvesting with a beam stiffener," Smart Materials and Structures, vol. 24, p. 032001, 2015.

[19] L. Zhao and Y. Yang, "An impact-based broadband aeroelastic energy harvester for concurrent wind and base vibration energy harvesting," Applied Energy, vol. 212, pp. 233-243, 2018.

[20] L. Zhang, H. Dai, A. Abdelkefi, and L. Wang, "Improving the performance of aeroelastic energy harvesters by an interference cylinder," Applied Physics Letters, vol. 111, p. 073904, 2017.

[21] G. Hu, K.-T. Tse, K. C. Kwok, J. Song, and Y. Lyu, "Aerodynamic modification to a circular cylinder to enhance the piezoelectric wind energy harvesting," Applied Physics Letters, vol. 109, p. 193902, 2016.

[22] L. Zhao and Y. Yang, "Analytical solutions for galloping-based piezoelectric energy harvesters with various interfacing circuits," Smart Materials and Structures, vol. 24, p. 075023, 2015.

[23] A. Bibo, A. Abdelkefi, and M. F. Daqaq, "Modeling and characterization of a piezoelectric energy harvester under Combined Aerodynamic and Base Excitations," Journal of Vibration and Acoustics, vol. 137, p. 031017, 2015.

[24] H. L. Dai, A. Abdelkefi, and L. Wang, "Piezoelectric energy harvesting from concurrent vortex-induced vibrations and base excitations," Nonlinear Dynamics, vol. 77, pp. 967-981, 2014.

[25] J. P. Den Hartog, Mechanical Vibrations. New York: McGraw-Hill, 1956.

[26] M. P. Païdoussis, S. J. Price, and E. De Langre, Fluid-structure interactions: Cross-flow-induced instabilities. New York: Cambridge University Press, 2010.

[27] A. Erturk and D. J. Inman, "On mechanical modeling of cantilevered piezoelectric vibration energy harvesters," Journal of Intelligent Material Systems and Structures, vol. 19, pp. 1311-1325, 2008. 